\title{
RF Displacement and Strain Sensing System for Wireless Structural Health Monitoring
}

\author{
Burak Ozbey*1, Ozgur Kurc ${ }^{2}$, Hilmi V. Demir' ${ }^{1}$, Vakur B. Erturk ${ }^{1}$, \\ and Ayhan Altintas ${ }^{1}$ \\ ${ }^{1}$ Dept. of Electrical and Electronics Engineering, Bilkent \\ University, TR-06800, Bilkent, Ankara, Turkey \\ ${ }^{2}$ Dept. of Civil Engineering, Middle East Technical University, \\ TR-06800, Ankara, Turkey
}

Structural health monitoring (SHM) is a technology with worldwide interest that is vital to ensure the reliability of any structure while also protecting the safety of human life. Over the years, a lot of research has been conducted on this topic, proposing SHM methods that may be instrumental in understanding the condition of critical parts of a structure. These methods generally rely on wired and/or active technologies, which are not preferable since the wires disallow telemetric measurements and mean increased weight and space, while the active technologies require electric power in order to operate. Two of the most important damage indices of a building structure such as a reinforcing bar (rebar) are the strain and displacement forming either with time or a sudden impact like an earthquake.

In this study, a wireless and passive RF sensing system that is able to monitor strain and displacement is presented. The system consists of two parts: A sensing unit which is attached on the structure to be monitored (e.g. a rebar or a piece of reinforced concrete) and an antenna which serves both as a transmitter and a receiver (B. Ozbey et al., Sensors, 14, 1691-1704, 2014). The sensing unit is developed as a comb-like nested split ring resonator (NSRR), a metamaterial-inspired structure whose resonance frequency changes by the number of its teeth pairs. The antenna is a microstrip single-slot antenna. The NSRR probe is coupled to the antenna in its near field; when a displacement forms along the monitored structural object, a local frequency peak or dip in the input impedance of this coupled system is shifted, and the sensor telemetrically detects these shifts.

It has been revealed by the experiments that the sensing system has a good resolution, responding to $\mu \mathrm{m}$-level changes. It exhibits a large measurement range of over $20 \mathrm{~mm}$, along with high levels of linearity $\left(R^{2}>0.99\right.$ over a range of $5 \mathrm{~mm})$ and sensitivity $\left(\mathrm{R}^{2}>12.7 \mathrm{MHz} / \mathrm{mm}\right.$ between $\left.1-3 \mathrm{~mm}\right)$. The sensor is able to function in both elastic and plastic deformation regions of steel when it is attached to a standard rebar (B. Ozbey et al., Sensors, 14, 19609-19621, 2014). During the presentation, results of both laboratory translation stage and high-force mechanical setup pulling experiments will be demonstrated. Additionally, new findings towards the employment of the sensor in fully reallife environment will be presented and the effects of building materials such as concrete and rebar mesh on the system performance will be discussed. 